\title{
A torsional MEMS varactor with wide dynamic range and low actuation voltage
}

\author{
C. Venkatesh ${ }^{\mathrm{a}, *}$, Shashidhar Pati ${ }^{\mathrm{a}}$, Navakanta Bhat ${ }^{\mathrm{a}}$, Rudra Pratap ${ }^{\mathrm{b}}$

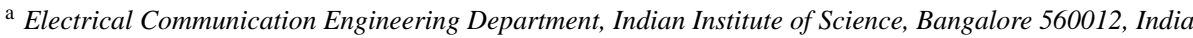 \\ ${ }^{\mathrm{b}}$ Mechanical Engineering Department, Indian Institute of Science, Bangalore 560012, India
}

\begin{abstract}
A MEMS varactor using torsional beams for actuation is proposed. Analytical expression for electrostatic torque developed is derived. The structure is compared with existing structures for dynamic range and actuation voltage. It is demonstrated that the proposed varactor outperforms the other structures in terms of wide dynamic range and lower actuation voltage, coupled with ease of fabrication. A methodology to choose the dimensions of varactor beams and plates has been outlined.
\end{abstract}

Keywords: Torsional MEMS varactor; Actuation voltage; Dynamic range

\section{Introduction}

Present day communication systems require high quality passive components to realize filters and voltage controlled oscillators. Varactors are an important component in many circuits. In IC technology a varactor is realized with reverse biased $\mathrm{p}-\mathrm{n}$ junction. Asymmetrical doping is required to achieve a large tuning range. But lower doping on one side results in large series resistance thereby reducing the quality factor. Hence there is a trade-off between dynamic range and quality factor. Also $Q$ varies with applied voltage. Thus passive components with very high quality-factor $(Q)$ are difficult to achieve in conventional IC technology. Hence components like inductors and varactors are currently off-chip, in the applications requiring high quality factor. Microelectromechanical systems have the potential to replace these off chip components and enhance the performance. In the recent past, many high- $Q$ MEMS varactors have been discussed in the literature [1-4]. Most of the problems with diode varactor

\footnotetext{
* Corresponding author. Tel.: +91 802932749; fax: +91 802932749

E-mail addresses: cvenkat@cedt.iisc.ernet.in, cvenkat@mems.ece.iisc.ernet.in (C. Venkatesh).
}

can be avoided with MEMS varactors. The main advantage of MEMS varactors is their high quality factor, which is crucial for use in frequency selection. Additionally MEMS varactors do not produce harmonic distortion as in the case of diode varactors. Tuning range, and low actuation voltage are the two main factors that are the focus of the current research in varactors.

Most MEMS varactors use electrostatic actuation due to favorable scaling of electrostatic force when compared to other actuation mechanisms. In recent past considerable effort has been directed towards achieving larger displacement and wider dynamic range using lower actuation voltage [2,5]. The tuning range of parallel plate varactor is limited due to the pull-in effect. Many structures are proposed to overcome this problem. Dec and Suyama [3] have used a three parallel plate configuration. The structure consists of two suspended plates and one fixed plate on the substrate. The fabrication process requires two layers of structural material resulting in three-plate arrangement. They were able to extend the tuning range from 50 to $87 \%$. Zou et al. [2] have proposed a wide tuning range varactor in which the gap between the varactor plates is $33 \%$ of the gap between the plates used for electrostatic actuation. This arrangement gives a tuning range of 
infinity. However the process integration is very complex requiring electrodes in three planes and hence dictating unequal sacrificial oxide removal.

To achieve low actuation voltage, a direct approach is to increase the length of levers (with meanders) and decrease the thickness of the levers [6]. This will result in consumption of large area.

This paper reports a novel MEMS varactor that uses torsion as a means of actuation. It is very simple to fabricate the structure, further the device can be either used as a threeor four-terminal varactor giving additional flexibility to the system designer.

\section{Proposed structure}

The proposed structure is shown in Fig. 1a and b. The structure consists of two sets of parallel plates. One of them is used for actuation and the other is used as the varactor. The actuating plate is connected to a torsional beam through another beam. The torsion beam is suspended with anchors at two ends. This arrangement creates a torque on the torsional beam when voltage is applied between the actuating plates. Hence the torsional beam undergoes twisting by a certain angle $\theta$. This angle is maximum at the center (point of application of torque) and zero near the anchor.

The varactor plates are attached to the torsion beam using two long levers. The plates are placed at a distance sufficiently away so that a small rotation of torsion beam results in a large displacement of the top plate of the varactor. Farther away the varactor plate is from the torsion beam the larger is the deflection for a given voltage.

Actuating plates can be added to both sides of the torsion beam, i.e. one more set of dc plates on the other side of the torsion beam. This arrangement can be used to create an upward force on ac plates. This force can be used to restore the ac plate to its normal position in case of stiction (between ac plates).

\section{Analysis and design}

It is evident that the torsion beam undergoes both torsion and bending. We need to know how much of the capacitance variation (in ac plate) is due to bending (and torsion) to accurately predict capacitance for a given voltage. To quantify

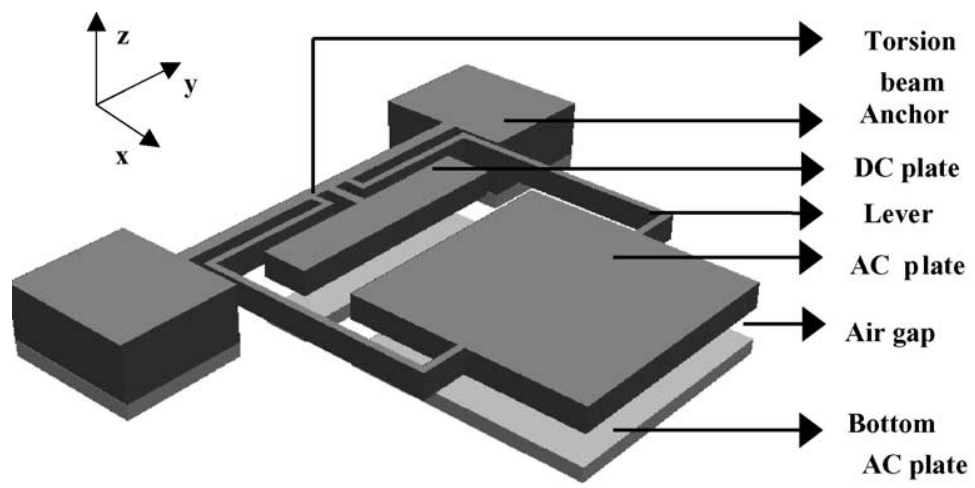

(a)

Isometric view of the proposed varactor

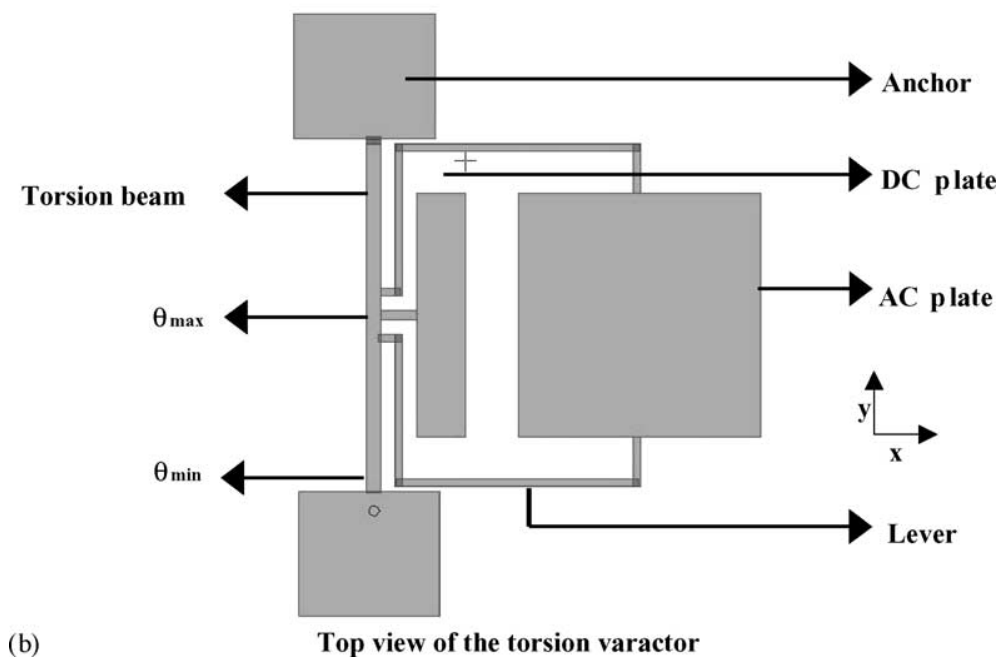

Fig. 1. (a) Isometric view of the proposed varactor. (b) Top view of the torsion varactor. 


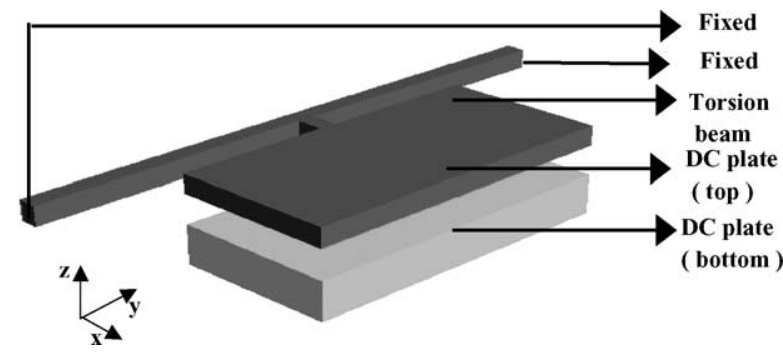

Fig. 2. Isometric view of the torsion beam with de plates.

Table 1

Dimensions of torsional structure

\begin{tabular}{lc}
\hline Part & Dimension microns \\
\hline Width of the beam & 4 \\
Thickness of beam and top plate & 0.5 \\
Length of moment arm & 15 \\
Width of dc plate (in $y$-direction) & 50 \\
Length of dc plate (in $x$-direction) & 20 \\
Air gap & 0.75 \\
\hline
\end{tabular}

Table 2

Percentage variation of linear displacement with length

\begin{tabular}{llll}
\hline Length microns & \multicolumn{2}{l}{ Displacement microns } & \\
\cline { 2 - 4 } & Linear & Torsional & Total \\
\hline 120 & $0.0055(27 \%)$ & 0.0145 & 0.02 \\
150 & $0.01(33 \%)$ & 0.02 & 0.03 \\
200 & $0.03(50 \%)$ & 0.03 & 0.06 \\
250 & $0.06(60 \%)$ & 0.04 & 0.10 \\
\hline
\end{tabular}

their relative contributions we carry out several simulations in Coventorware with varying torsion beam length. The structure shown in Fig. 2 is used for the simulations.

Simulations were carried out with dc plate and torsion beam alone, as varactor (ac) plate does not play any role in bending or twisting of torsion beam. Table 1 gives the dimensions of the structure used for the simulation. A voltage of $0.5 \mathrm{~V}$ is applied between the plates. Torsion beam length is varied from 100 to $250 \mu \mathrm{m}$. Table 2 gives the length of the beam and corresponding displacement at the farthest edge of dc plate (parallel to beam). The bending and torsion components of displacement are extracted from simulation results. The bending component is essentially the displacement of torsion beam as shown in Fig. 3. As the beam bends down the whole of dc plate (as well as ac plate, if it is present) also moves down by the same amount. In torsion the beam twists and farthest edge of dc plate experiences largest downward displacement, i.e. "torsional displacement" gets amplified whereas "linear displacement" does not get amplified. If the varactor has been designed taking into account only torsion, it would lead to pull-in of the dc plate before the ac plate has spanned the total distance " $d$ ", due to linear displacement. In that case capacitance would change abruptly. Hence the linear displacement should be considered in designing torsional varactor, especially if the length of the torsion beam is long.

In analyzing the structure, bending and twisting forces were decoupled and treated separately. When the voltage is applied on the dc plate, a distributed force develops throughout the plate. This is shown schematically in Fig. 4a. This distributed force can be integrated to get resultant lumped force. If we have point force acting at a distance from a support we can replace it with a couple $(M)$ and a force $(V)$ (Fig. $4 \mathrm{~b}$ and c). Now we can analyze the beam with a decoupled force and a couple and derive "bending expressions" and "torsion expressions" separately.

The expression for electrostatic field at a point $x$ (Fig. 5) on the dc plate is [7]

$E=\frac{V}{a}=\frac{V}{((d / \sin \theta)-x) \theta}$

where $V$ is the applied voltage. Electrostatic force per unit area is given by the expression,

$P=\frac{\varepsilon E^{2}}{2}$

where $\varepsilon=8.85 \times 10^{-12} \mathrm{~F} / \mathrm{m}$. Total force can be obtained by integrating this expression over dimensions of the dc plate.

$F_{\mathrm{T}}=\frac{V^{2} W \varepsilon}{2} \int_{L_{1}}^{L_{2}} \frac{\mathrm{d} x}{((d / \sin \theta)-x)^{2} \theta^{2}}$
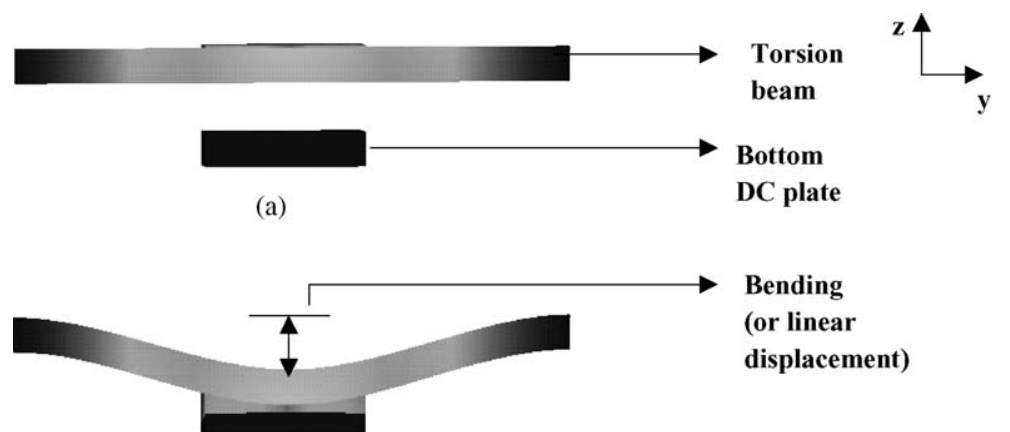

(b)

Fig. 3. (a) Torsion beam without bending. (b) Torsion beam with bending. 


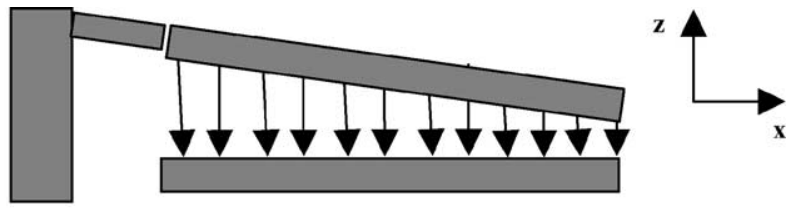

(a) Schematic showing the distributed electrostatic force (side view)

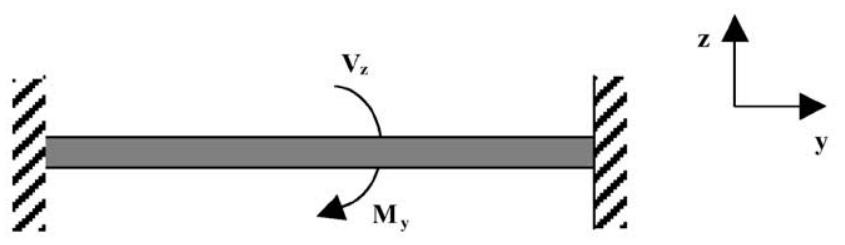

(b) Distributed force replaced by a force (V) and a moment (M)

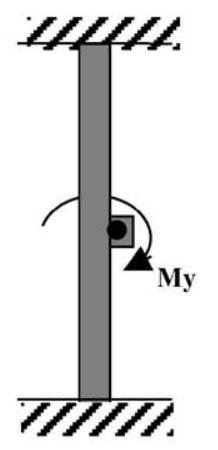

(c) Top-view

Fig. 4. (a) Schematic showing the distributed electrostatic force (side view). (b) Distributed force replaced by a force $(V)$ and a moment $(M)$. (c) Top-view.

$F_{\mathrm{T}}=\frac{V^{2} W \varepsilon}{2 \theta^{2}}\left[\frac{1}{\left((d / \sin \theta)-L_{2}\right)}-\frac{1}{\left((d / \sin \theta)-L_{1}\right)}\right]$

Similarly total torque can be obtained by multiplying the force with moment arm " $x$ ".

$T_{\mathrm{E}}=\frac{V^{2} W \varepsilon}{2} \int_{L_{1}}^{L_{2}} \frac{x \mathrm{~d} x}{((d / \sin \theta)-x)^{2} \theta^{2}}$

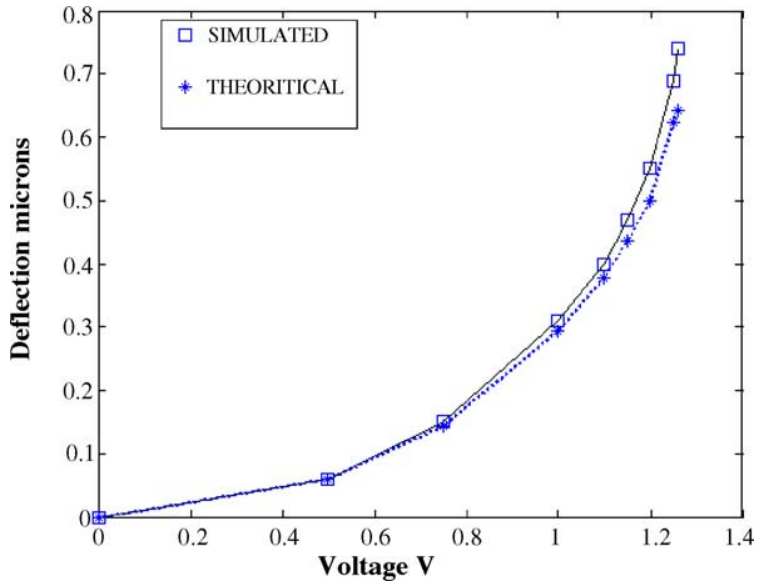

Fig. 6. Simulated vs. theoretical prediction of deflection.

$=\frac{\varepsilon V^{2} W}{2 \theta^{2}}\left[\log \left(\frac{d-L_{2} \theta}{d-L_{1} \theta}\right)+d\left(\frac{1}{d-L_{2} \theta}-\frac{1}{d-L_{1} \theta}\right)\right]$

Formulas are available for bending (linear) spring constant [4] and torsional spring constant of a fixed-fixed beam $\left(L_{\text {beam }}\right.$ in our case). The formula for torsional spring constant is $[7,8]$

$K_{\theta}=\frac{2 G w t^{3}}{3 L_{\text {beam }}}\left[1-\frac{192 t}{\pi^{5} w} \tanh \left(\frac{\pi w}{2 t}\right)\right]$

and spring constant for bending is

$K_{\mathrm{b}}=16 E w\left(\frac{t}{L_{\text {beam }}}\right)^{3}$

Dividing (4) by (8) would give us the deflection at the middle of the beam. Similarly (6) and (7) would yield the rotation angle $\theta$. It can be seen that $K_{\mathrm{b}}$ varies as $1 / L_{\text {beam }}^{3}$ and $K_{\theta}$ varies and $1 / L_{\text {beam. }}$. So if the beam is longer bending dominates.

Eqs. (1)-(8) are used to calculate the deflection at the edge of ac plate as the voltage is increased. Fig. 6 shows the graph of voltage versus deflection. It is seen that theoretical value matches well with the simulated value. As the deflection increases the error increases. This can be attributed to the fringing fields. Simulations predict a pull-in voltage of $1.27 \mathrm{~V}$ whereas theoretical calculations predict pull-in at $1.35 \mathrm{~V}$.
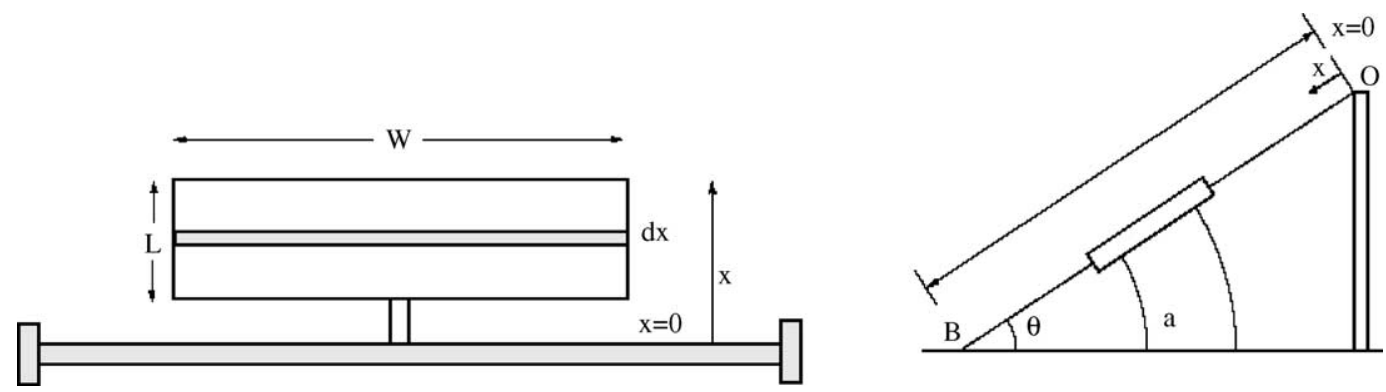

Fig. 5. Top-view of dc plate. 


\section{Optimal dimensions}

In this section we will outline a procedure to determine the optimal dimensions of various beams in torsional varactor. Dimensions of ac plate are decided by the required capacitance. Dimensions of dc plate depend on voltage available for actuation (say 0-5 V).

For a given area, the dynamic range $\left(C_{\max } / C_{\min }\right)$ would be more for an ac plate that is longer along $y$-direction than $x$. This can be established analytically as follows.

The expression for capacitance can be derived by integrating per-unit area capacitance all over the ac plate. Let the given area be " $A$ ". Let the length be " $L$ " $(x$-direction) and the width " $W$ " ( $y$-direction) be " $L / n$ " where " $n$ " is a constant. We have

$A=\left(\frac{L}{n}\right) L=\frac{L^{2}}{n}$

Let the constant $n$ be greater than one, i.e. width is less than length. The expression for capacitance is

$C=\varepsilon W \int_{L_{1}}^{L_{2}} \frac{\mathrm{d} x}{((d / \sin \theta)-x) \theta}$

For small angle of rotation, $\sin \theta \approx \theta$. Therefore we obtain,

$C=\varepsilon \frac{W}{\theta} \log \left(\frac{d-L_{1} \theta}{d-L_{2} \theta}\right)$

For the present analysis let $L_{1}=0$. So we have

$C=\varepsilon \frac{W}{\theta} \log \left(\frac{d}{d-L_{2} \theta}\right)$

We consider the following two cases:

1. length $=L$, width $=L / n, n>1$;

2. length $=L / n$, width $=L, n>1$.

$C_{\min }$ (for voltage $V=0$ ) for both cases is equal, since the top plate remains parallel to the bottom plate. As the voltage

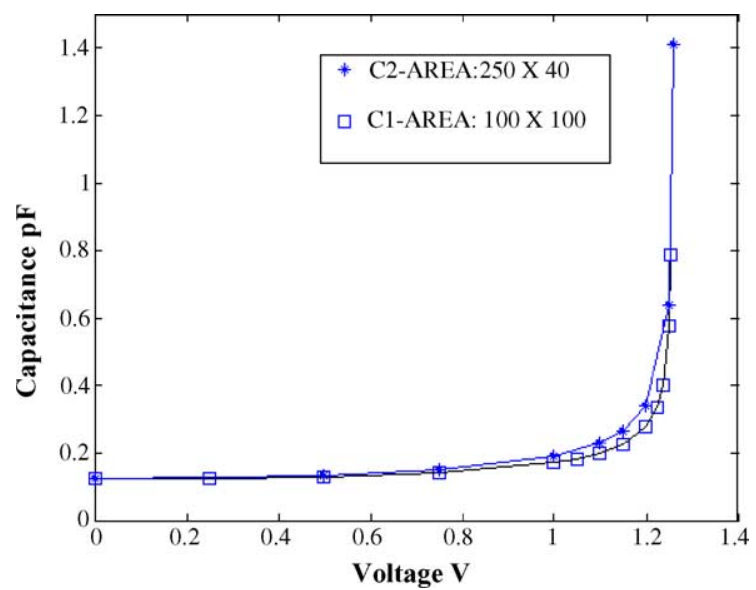

Fig. 7. Comparison between capacitors with different shape.

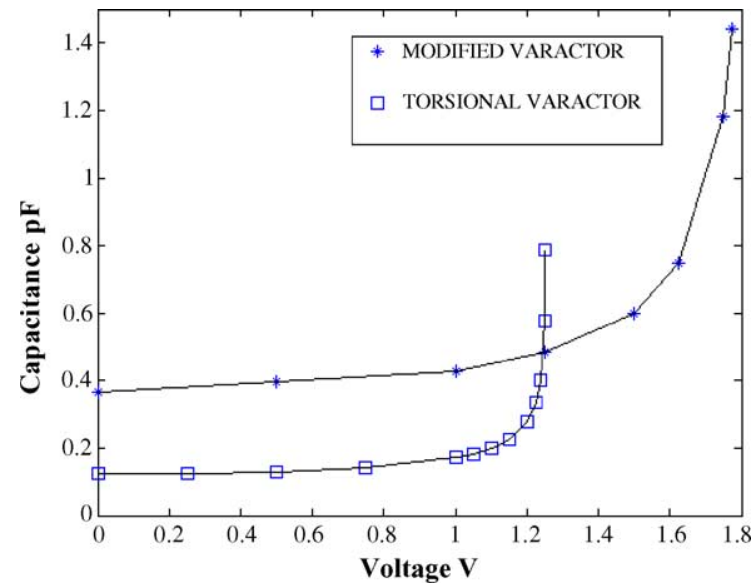

Fig. 8. Comparison between torsional varactor and modified varactor.

is applied $\theta$ increases and the expression (5) applies (It does not hold for $\theta=0$ ). We have two different expressions for the two cases considered

Case 1: $\quad C_{1}=\varepsilon\left(\frac{L}{n \theta}\right) \log \left(\frac{d}{d-L \theta}\right)$

Case 2: $\quad C_{2}=\varepsilon \frac{L}{\theta} \log \left(\frac{n d}{n d-L \theta}\right)$

Taking ratio of $C_{1}$ and $C_{2}$ we have,

$\frac{C_{2}}{C_{1}}=\frac{n \log (n d /(n d-L \theta))}{\log (d /(d-L \theta))}=n \frac{\log (n d)-\log (n d-L \theta)}{\log (d)-\log (d-L \theta)}$

Numerator of the ratio can be manipulated as follows

$$
\begin{aligned}
& \log (n d)-\log (n d-L \theta)>\log (n d)-\log (n d-n L \theta) \\
& \quad=\log (n)+\log (d)-\log (n)-\log (d-L \theta) \\
& \quad=\log (d)-\log (d-L \theta)
\end{aligned}
$$

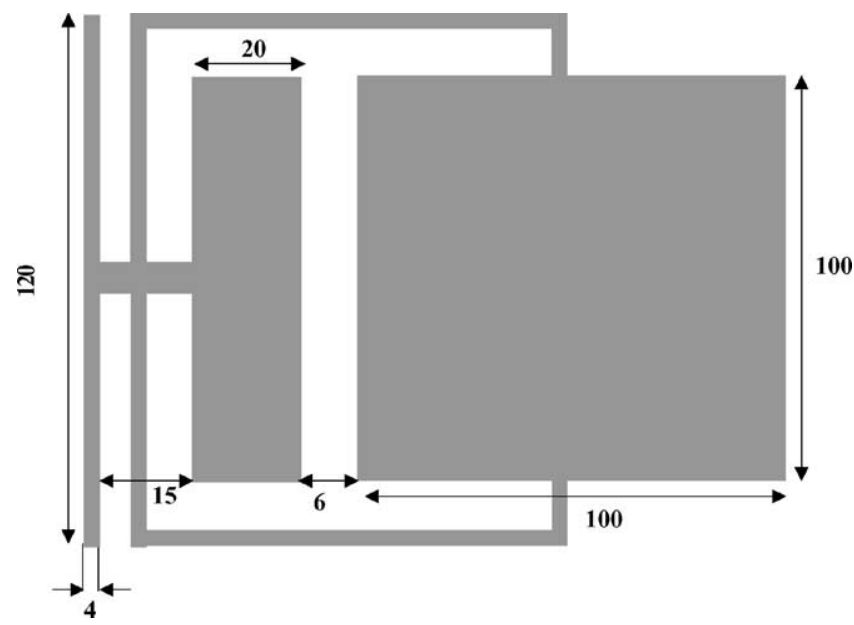

Fig. 9. Top-view of torsion varactor with dimensions (all dimensions are in micrometers). 
Substituting this in the expression for $C_{2} / C_{1}$ we get,

$$
\begin{aligned}
\frac{C_{2}}{C_{1}}= & n \frac{\log (C d)-\log (C d-L \theta)}{\log (d)-\log (d-L \theta)} \\
& >n \frac{\log (d)-\log (d-L \theta)}{\log (d)-\log (d-L \theta)}=n
\end{aligned}
$$

Hence we have $C_{2}>C_{1}$.

Simulations are done with two different shapes of ac plate. Fig. 7 shows the results. It is seen that capacitor that is longer along $y$-direction (parallel to torsion beam) gives higher capacitance for same voltage and also higher dynamic range.

In designing the dc plate we have many parameters to manipulate. A particular dynamic range in a given voltage range can be obtained with different combinations of dimensions, i.e. there is no unique value for the lengths and widths of the structure. Our aim is to maximize $\theta$ for a given voltage. Expressions (1)-(8) can be used to get quantitative idea of variation of $\theta$ for a unit variation in dimensions of the

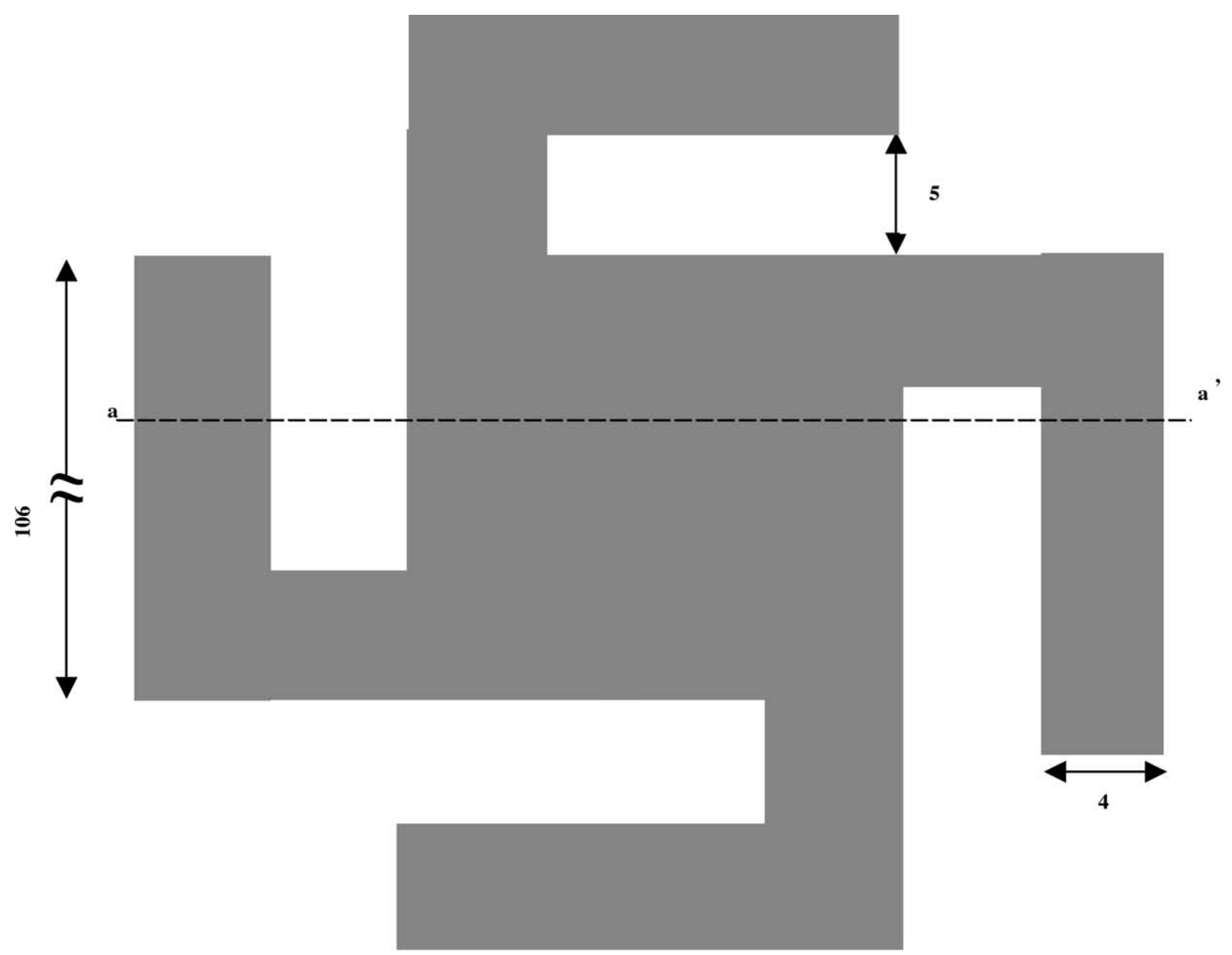

(a)

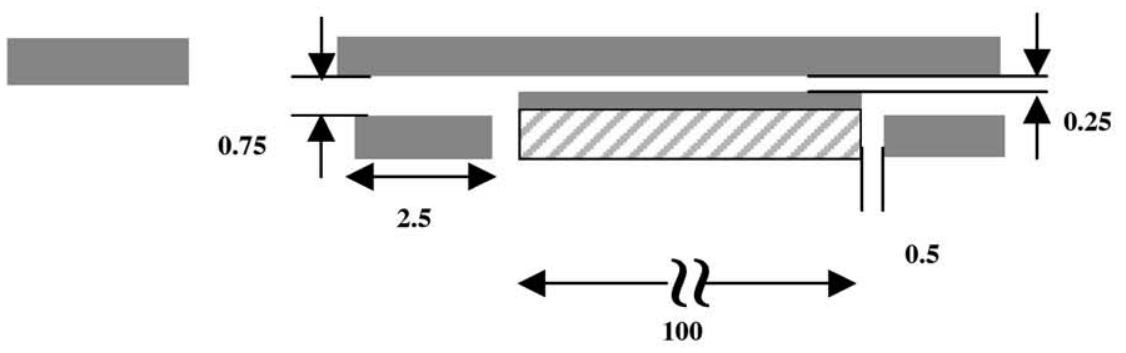

(b)

Fig. 10. (a) Top view of modified conventional varactor. (b) Side view along $a-a^{\prime}$ plane. 
structures. An algorithm can be used to decide the direction along which the increment should be made. Thus design of dc plate is an optimization problem.

\section{Simulation}

Simulation studies are done for torsional varactor using Coventorware. Fig. 8 shows the capacitance variation with the applied voltage, and it can be seen that a very low actuation voltage is sufficient to get the large dynamic range. The results are compared with an existing structure, the modified conventional varactor [2], under the constraint that total area occupied by all the structures is same $\left(\approx 17,000 \mu \mathrm{m}^{2}\right)$. Dimensions of torsional varactor and modified varactor are given in Figs. 9 and 10, respectively. The results are shown in Fig. 8. Torsional varactor spans the gap of $0.75 \mu \mathrm{m}$ in $1.25 \mathrm{~V}$ and modified parallel plate varactor requires $1.8 \mathrm{~V}$. Both give a comparable dynamic range. The superior performance of torsional varactor is evident from this comparison. Further, the torsional varactor requires the electrode plates only in two planes as opposed to modified conventional varactor and can be realized through standard PolyMUMPS process [9].

\section{Conclusions}

The proposed torsional varactor has superior performance compared to the existing structures. The large dynamic range at low actuation voltage has been verified with rigorous electromechanical simulations using Coventorware. An analytical model for electrostatic torque has been derived. A procedure has been outlined for selecting optimal dimensions of varactor beams and plates.

\section{Acknowledgements}

The authors like to acknowledge the support received from National Program on Smart Materials (NPSM). This work was carried out at National MEMS design Centre funded by NPSM.

\section{References}

[1] Z.X. Xiao, W.Y. Peng, R.F. Wolffenbuttel, K.R. Farmer, Micromachined variable capacitors with wide tuning range, Sens. Actuators A 104 (2003) 299-305.

[2] J. Zou, C. Liu, J. Schutt-Aine, J.H. Chen, S.M. Kang, Development of a wide tuning range MEMS tunable capacitor for wireless communication systems, Tech. Digest, IEEE Int. Electron Devices Meet, December 10-13, 2000, San Francisco, USA, pp. 403-406.

[3] A. Dec, K. Suyama, Micromachined electro-mechanically tunable capacitors and their applications to RF IC's, IEEE Trans. Microwave Theory Tech. 46 (1998) 2587-2596.
[4] G.M. Rebeiz, Rf MEMS Theory, Design and Technology, WileyInterscience, New Jersey, 2003.

[5] E.S. Hung, S.D. Senturia, Extending the travel range of analogtuned electrostatic actuators, IEEE J. Microelectromech. Sys. 8 (1999) 497-505.

[6] S.P. Pacheco, L.P.B. Katehi, C.T.C. Nguyen, Design of low actuation voltage RF MEMS switch, Microwave Symposium Digest, IEEE MTT-Symposium, June 11-16, 2000, Boston, USA, pp. 165168.

[7] H. Toshiyoshi, H. Fujita, Electrostatic micro torsion mirrors for an optical switch matrix, IEEE J. Microelectromech. Sys. 4 (1996) 231-237.

[8] S.P. Timoshenko, J.N. Goodier, Theory of Elasticity, 3rd ed., McGrawHill, New York, 1970.

[9] MUMPS Design Handbook, Revision 5.0, Cronos Integrated Microsystems, Research Triangle Park, NC, USA, 2000.

\section{Biographies}

C. Venkatesh received his undergraduate degree in Instrumentation and Control engineering from Regional Engineering College (REC), Tiruchirapalli, Tamil Nadu, India, in 1998. Subsequently, he received his M.Sc. (Engg.) degree in Electrical Engineering from Indian Institute of Science. At present he is working towards his Ph.D. at Indian Institute of Science. His research interests include RF MEMS, microelectronic circuits and system dynamics and control.

Shashidhar Pati received his B.E. degree in Electrical and Electronics Engineering from N.B.K.R. Institute of Technology, Andhra Pradesh, India in 2001 and M.E. degree in Microelectronics from Indian Institute of Science, Bangalore, India, in the year 2003. Currently he is working as design engineer for Alliance Semiconductor, India. His research interests are RF MEMS and clock syntheses (PLL/DLL) circuits.

Navakanta Bhat received his B.E. in Electronics and Communication from University of Mysore in 1989, M.Tech. in Microelectronics from I.I.T., Bombay, in 1992 and Ph.D. in Electrical Engineering from Stanford University, Stanford, CA, in 1996. Then he worked at Motorola's Networking and Computing Systems Group in Austin, TX, until 1999. At Motorola he worked on logic technology development and he was responsible for developing high performance transistor design and dual gate oxide technology. He joined the Indian Institute of Science, Bangalore, in 1999 where he is currently an Associate Professor in the Electrical Communication Engineering Department. His current research is focused on Analog and RF Microsystems using CMOS and MEMS technology. The work includes process development, device design and modeling, circuit design. He has several research publications in international journals and conferences and two US patents to his credit. He is the recipient of the Young Engineer Award (2003) from the Indian National Academy of Engineering. He was the founding chair of the IEEE Electron Devices and Solid-State Circuits Society, Bangalore Chapter which was recognized as the Outstanding Chapter of the Year (2003) by the IEEE SSC society. He has been on the program committees of several international conferences, including the ISSCC.

Rudra Pratap received his B.Tech. degree from I.I.T., Kharagpur, in 1985, M.S. from University of Arizona, Tucson, in 1987, and $\mathrm{Ph} . \mathrm{D}$. from Cornell University, Ithaca, NY, in 1993. He specialized in engineering mechanics. After finishing his Ph.D. in the area of nonlinear dynamics, he taught in the Sibley School of Mechanical and Aerospace Engineering at Cornell University till 1996. In August 1996, he joined the Department of Mechanical Engineering at the Indian Institute of Science, Bangalore, where he is currently an Associate Professor. His current research focus is on MEMS design and phenomenological studies in dynamic response of micro structures and devices. Other than those on MEMS, he has published several papers in the areas of nonlinear 
dynamics, bifurcation theory, and chaos, and remains keenly interested in the studies of nonlinear mechanics. He also has two books to his credit, one a popular starter book on MATLAB and one on engineering mechanics, co-authored with Prof. Andy Ruina, Cornell University. The book on mechanics is still in beta version and available for class tests.
He is also on the Editorial Board of CMC: Computers, Materials, and Continua, an international journal from Tech Science Press. He currently heads the CranesSci MEMS Lab at I.I.Sc., which is the only MEMS research Lab in India established with participation from a private company. 